\title{
RIS-Aided Wireless Communications: Prototyping, Adaptive Beamforming, and Indoor/Outdoor Field Trials
}

\author{
Xilong Pei, Haifan Yin, Li Tan, Lin Cao, Zhanpeng Li, Kai Wang, Kun Zhang, and Emil Björnson
}

\begin{abstract}
The prospects of using a Reconfigurable Intelligent Surface (RIS) to aid wireless communication systems have recently received much attention from academia and industry. Most papers make theoretical studies based on elementary models, while the prototyping of RIS-aided wireless communication and real-world field trials are scarce. In this paper, we describe a new RIS prototype consisting of 1100 controllable elements working at $5.8 \mathrm{GHz}$ band. We propose an efficient algorithm for configuring the RIS over the air by exploiting the geometrical array properties and a practical receiver-RIS feedback link. In our indoor test, where the transmitter and receiver are separated by a $30 \mathrm{~cm}$ thick concrete wall, our RIS prototype provides a 26 dB power gain compared to the baseline case where the RIS is replaced by a copper plate. A $27 \mathrm{~dB}$ power gain was observed in the short-distance outdoor measurement. We also carried out long-distance measurements and successfully transmitted a 32 Mbps data stream over $500 \mathrm{~m}$. A 1080p video was live-streamed and it only played smoothly when the RIS was utilized. The power consumption of the RIS is around $1 \mathrm{~W}$. Our paper is vivid proof that the RIS is a very promising technology for future wireless communications.
\end{abstract}

Index Terms-Reconfigurable intelligent surface, intelligent reflecting surface, RIS, IRS, LIS, metasurface, metamaterial, MIMO, massive MIMO, prototype, field trials.

\section{INTRODUCTION}

Massive multiple-input multiple-output (MIMO) technology $[1]-[3]$ is at the core of the 5th generation (5G) cellular communication technology. The base stations make use of arrays of 64 or more antenna-integrated radios [4] to enable precise beamforming towards any location in the cell and to enable spatial multiplexing of many user terminals. The technology can focus energy in space but it cannot overcome major weaknesses imposed by the propagation environment, such as shadow fading. The losses by shadowing particularly evident when the carrier frequency increases, e.g., from the 3.5 $\mathrm{GHz} 5 \mathrm{G}$ band to millimeter wave (mmWave) bands, and even towards the (sub-)terahertz bands. The traditional approach to overcome such propagation limitations is to utilize relays in between the base station and intended receivers to fill coverage

X. Pei, H. Yin, L. Tan, L. Cao, Z. Li, K. Wang, K. Zhang are with the School of Electronic Information and Communications, Huazhong University of Science and Technology, Wuhan, China. E-mail: \{pei, yin, ltan, caolin, zhanpli, kaiw, zkun\}@ hust.edu.cn.

E. Björnson is with the KTH Royal Institute of Technology, Sweden, and Linköping University, Sweden. E-mail: emilbjo@kth.se.

The corresponding author is Haifan Yin.

This work was supported by the National Natural Science Foundation of China under Grant 62071191. E. Björnson was supported by the FFL18-0277 grant from the Swedish Foundation for Strategic Research. holes [5]. This equipment could be anything from passive repeaters (e.g., a carefully rotated copper plate that reflects signals in a predetermined direction) to relays with baseband processing (e.g., using a decode-and-forward protocol where an amplified signal is retransmitted after noise removal).

A new type of relaying technology has appeared inspired by recent advances in electromagnetic metamaterials [6]-[9]. It is known as a Reconfigurable Intelligent Surface (RIS) [10] or Intelligent Reflecting Surface [11]. In a nutshell, an RIS is a two-dimensional surface that can be electronically tuned to interact with electromagnetic waves as if it had another physical shape; for example, if one wants the transmitted signal to be reflected towards a certain location, the RIS can synthesize a metal plate that is rotated and bent to focus the incident waves on that location [12]. Practically speaking, an RIS can be built using artificial electromagnetic metamaterial, which consists of periodic arrangements of specifically designed subwavelength-sized structural elements [13]. Such metamaterials have unique electromagnetic properties that do not exist in nature [14], such as, negative refraction [15], perfect absorption, and anomalous reflection/scattering [16]. In order to realize the aforementioned relaying feature, the RIS must contain a large number of elements (to obtain appreciable gains) that have controllable properties [17]. By varying the reflection coefficient (e.g., phase shift) of the elements, one can in theory control towards which location an incident wave is beamformed. The number of discrete states that an element can have is often measured in bits, because an element with $2^{N}$ states can be controlled using $N$ bits [18].

We will put the concept of RIS to practice by designing an RIS-enhanced wireless communication prototype and using it for indoor/outdoor field trials. A previous prototype was described in $[19]-[21]$. It was designed to modulate the impinging signal, which is very different from our work, where we passively reflect/relay information-carrying wireless signals towards the receiver. In [22], an RIS prototype with 256 2-bit elements was presented. It achieved a $21.7 \mathrm{dBi}$ antenna gain for a fixed configuration and no adaptive beamforming was reported. The power consumption was around $153 \mathrm{~W}$. [23] proposed RFocus, which is a system that moves beamforming functions from the radio transmitter to the environment. Their measurements showed an improvement in median signal strength by 9.5 times and doubled the median channel capacity. The beamforming functionality relied on signal strength measurements, which is the Received Signal Strength Indication (RSSI). In [24], Metawave Corporation demonstrated a 5G 
coverage extension use case with a $28 \mathrm{GHz}$ passive reflector.

In this paper, we describe a prototype of RIS-aided wireless communication system that can serve mobile users through feedback-based real-time beamforming at the RIS. Our system operates in the $5.8 \mathrm{GHz}$ band, which is the unlicensed band used by wireless local area network (WLAN). The host computer of the transmitter encodes a video stream to be transmitted. The transmitter carries out baseband signal processing and radio-frequency (RF) signal transmission through a horn antenna. The RIS controls the reflection coefficients by adjusting the impedance of the patches and thereby beamforming the impinging signal towards the receiver. The receiver processes the baseband signal and demodulates the video stream. In the meantime, the receiver calculates the strength of the received signal and feeds back this information to the RIS. The RIS updates the reflection coefficients according to a proposed selfadaptive beamforming algorithm. The power consumption of our RIS is only about $1 \mathrm{~W}$.

The main contributions of our work are as follows:

- We have developed a prototype for RIS-aided communication and evaluated its basic properties in the lab.

- We verify the uplink and downlink channel reciprocity of the RIS communication channel. We also demonstrate the reflection phase response for a given control voltage varies with the incident angle of the electromagnetic wave. This non-ideal feature must be considered in practical designs.

- We have proposed and tested a novel feedback-based adaptive reflection coefficient optimization algorithm, which enables smart reflection without having to modify the existing communication standards.

- We conduct the world's first indoor test without a Lineof-Sight (LoS) path and demonstrate a $26 \mathrm{~dB}$ power gain, compared to having a copper plate that lacks the capability of smart reflection.

- We conduct the world's first long-range outdoor test. In the $50 \mathrm{~m}$ and $500 \mathrm{~m}$ field trials, the RIS achieved up to $27 \mathrm{~dB}$ power gains. In the longest scenario, a $32 \mathrm{Mbps}$ data rate was achieved with the help of the RIS.

The rest of this paper is organized as follows. Section II describes the details of our RIS design. In Section III], we show the prototype of an RIS-based wireless communication system. In Section IV] we focus on the design of the reflection coefficients. Then in Section $\mathrm{V}$, we make several experiments to verify the system performance. Finally, the conclusions are drawn in Section VI

\section{Design of Reconfigurable Intelligent Surface}

In this section, we describe the details of our new large-scale RIS hardware prototype, which consists of 1100 elements.

\section{A. Reflective Element Design}

We designed a metasurface tuned by varactor diodes for operation in the C-band. Fig. 1 shows a varactor diode tuned structure. The structure of the designed reflective element is inspired by [25], with an adaption to the desired frequency. The structural parameters are listed in Table I and their

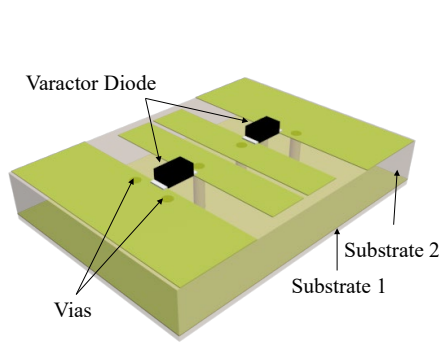

(a) Perspective view

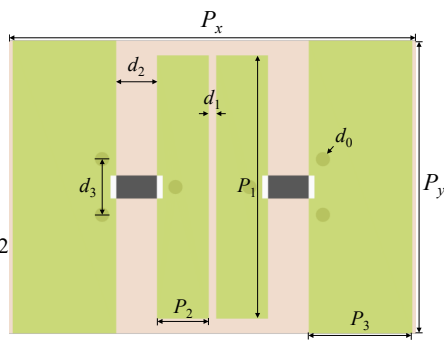

(b) Top view

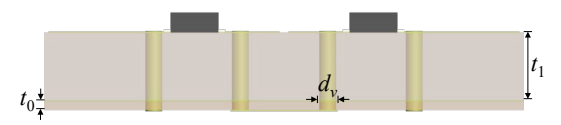

(c) Side view

Fig. 1. The topology of an RIS element. Each element contains two varactor diodes, and the parameters are marked in the figure.

TABLE I

The Structural Parameters of AN RIS ElEment

\begin{tabular}{|c|c|}
\hline \hline Parameter & Value \\
\hline Period & $P_{x}=14.3 \mathrm{~mm}, P_{y}=10.27 \mathrm{~mm}$ \\
\hline Rectangular patch size & $P_{1}=9.23 \mathrm{~mm}, P_{2}=1.82 \mathrm{~mm}, P_{3}=3.64 \mathrm{~mm}$ \\
\hline Patch slot width & $d_{1}=0.26 \mathrm{~mm}, d_{2}=1.43 \mathrm{~mm}$ \\
\hline Via-holes clearance & $d_{3}=1.95 \mathrm{~mm}$ \\
\hline Ground hole opening & $d_{v}=0.6 \mathrm{~mm}$ \\
\hline Via diameter & $d_{0}=0.5 \mathrm{~mm}$ \\
\hline Substrate 1 & FR4 $\left(\varepsilon_{r}=4.4, \tan \delta=0.02, t_{0}=0.254 \mathrm{~mm}\right)$ \\
\hline Substrate 2 & F4B $\left(\varepsilon_{r}=2.65, \tan \delta=0.005, t_{1}=2 \mathrm{~mm}\right)$ \\
\hline \hline
\end{tabular}

meanings are shown in Fig. 1] The reflective elements are densely packed without spacing. Each element consists of three layers. The top layer contains two pairs of rectangular metallic patches, each of which is connected by a varactor diode with the junction capacitance controlled by the external bias voltage. The middle layer is a ground plane for reflecting impinging waves. The ground plane is connected to the patches on both sides of the top layer through four via-holes serving as ground, for design convenience. The bottom layer contains direct current (DC) biasing lines that regulate the varactor diodes on the top layer. Two via-holes connect the central patches to biasing lines. The copper thickness of the patches and biasing lines of the elements are all $35 \mu \mathrm{m}$. There are two kinds of substrates between the three layers, which are FR4 and F4B. FR4 is a composite material composed of woven fiberglass cloth with an epoxy resin binder. F4B is a series of high frequency materials, which are cheap in price and stable in quality.

The properties of the proposed element were first evaluated with the commercial electromagnetic solver CST Microwave Studio 2019, using periodic boundary conditions to quantify the reflection spectra for different bias voltages. The varactor diodes integrated into the element are Skyworks SMV2019079LF, which can be modeled as a series Resistor-InductorCapacitor (RLC) circuit (i.e., it consists of a resistance, a capacitance, and an inductance connected in series) as shown in Fig. 2. The equivalent circuit component parameters are shown 


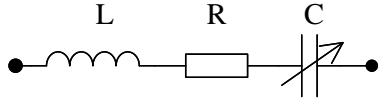

Fig. 2. An equivalent circuit description of the varactor diode. It can be modeled as a series RLC circuit.

TABLE II

Equivalent Circuit Parameters of VARACTOR Diode [26]

\begin{tabular}{|cccc|}
\hline \hline VR $(\mathbf{V})$ & $\mathbf{C}(\mathbf{p F})$ & $\mathbf{R}(\mathbf{o h m})$ & $\mathbf{L}(\mathbf{n H})$ \\
\hline 0 & 2.31 & 4.51 & 0.70 \\
\hline-4 & 0.84 & 4.04 & 0.70 \\
\hline-7 & 0.55 & 3.66 & 0.70 \\
\hline-11 & 0.38 & 3.18 & 0.70 \\
\hline-14 & 0.31 & 2.86 & 0.70 \\
\hline-16 & 0.27 & 2.65 & 0.70 \\
\hline-19 & 0.24 & 2.38 & 0.70 \\
\hline \hline
\end{tabular}

in Table II] where "VR" denotes the Reverse Voltage applied on the diode. The varactor diode is modeled by a lumped port in the simulation. The amplitude and phase responses of the reflection under different bias voltages are shown in Fig. 3 The observed behavior is aligned with the canonical case described in [12], where different voltages result in widely different phase shifts over the considered frequency range. For any given frequency within the yellow-marked band from 5.5 $\mathrm{GHz}$ to $6.0 \mathrm{GHz}$, varying the bias voltage from 0 to $19 \mathrm{~V}$ is sufficient to modify the phase-shift by at least 180 degrees. The amplitude variations are substantially smaller within the considered band and the largest losses appear when the phase is close to zero.

We have designed a 1-bit prototype where the two states have a phase difference of around 180 degrees. Note that this granularity is sufficient to configure a set of elements so their reflected signals are (partial) coherently combined at a location of choice [18]. The orange region in the middle of Fig. 3 represents a $20 \mathrm{MHz}$ bandwidth around the central frequency of $5.8 \mathrm{GHz}$. We can conclude from the figure that the considered element structure can achieve a 180 degree phase difference in this region, for example, using $7 \mathrm{~V}$ and $16 \mathrm{~V}$ as bias voltages, for which the phase shifts are around -90 and +90 degrees, respectively. Moreover, the reflection coefficients are approximately constant within the considered bandwidth, thus the RIS element has a frequency-flat response.

\section{B. RIS Board Design}

The RIS board consists of a multitude of RIS elements and their controllers. In [27], an RIS that can provide continuous phase shift variations for each element was proposed. Its micro-controllers generate Pulse-Width Modulation (PWM) signals that are low-pass filtered to create bias voltages that can be continuously varied from 0 to $5 \mathrm{~V}$. In this method, the PWM signal controlling each element needs to be generated independently, and a corresponding low-pass filter circuit is needed. Thus, the cost and hardware complexity will increase dramatically when building a large-scale RIS. A high phaseshift resolution might be useful when using an RIS to cancel

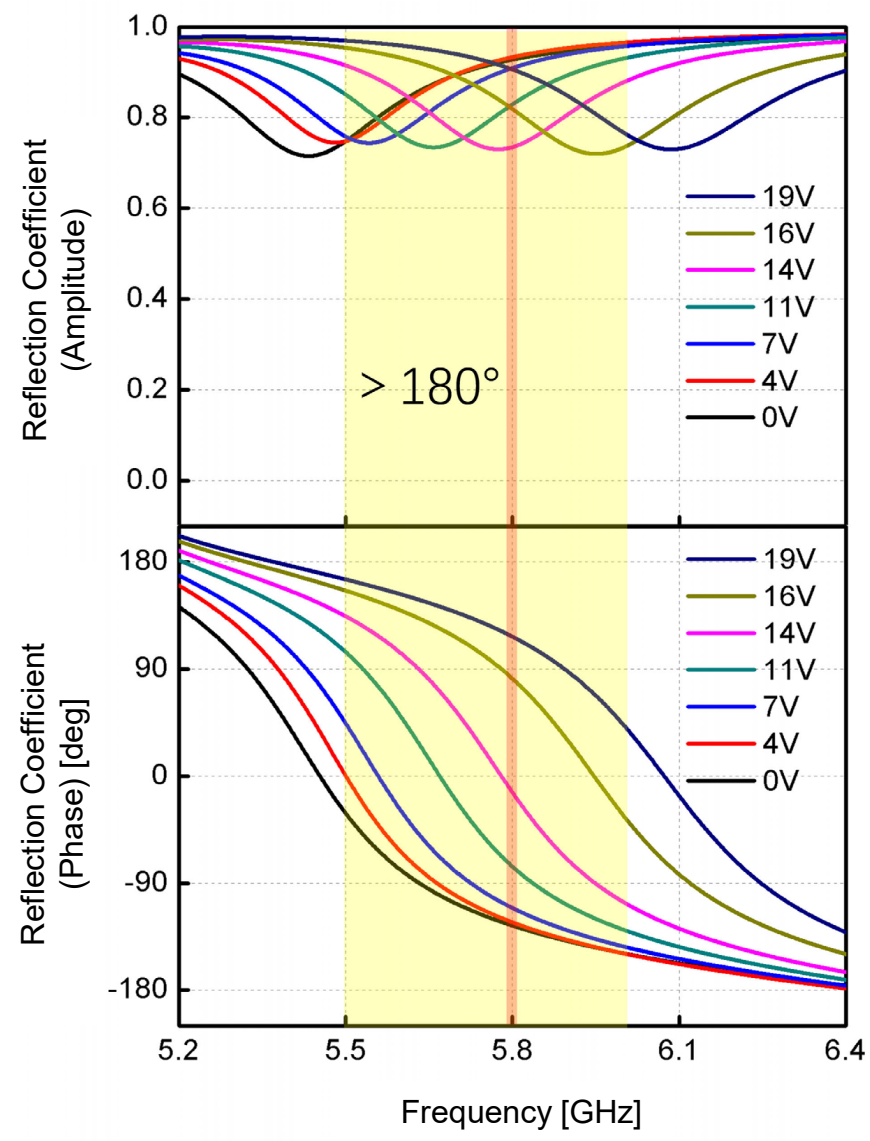

Fig. 3. The simulated reflection amplitude and phase response of the RIS element under different voltages from 0 to $19 \mathrm{~V}$. The yellow region represents the range where the maximum phase difference is larger than 180 degrees. The orange region will be used for communications in the field trials.

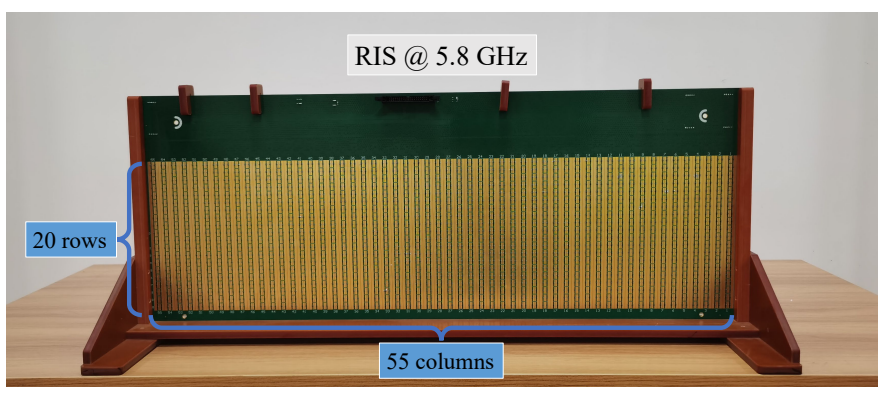

Fig. 4. The front view of the fabricated $55 \times 20$ RIS, each element capable of 1-bit phase shifting. The size is $80.08 \mathrm{~cm} \times 31.30 \mathrm{~cm}$.

interference in multi-user scenarios [11], but the goal of our field trials is for the RIS to reflect the incident waves as a beam pointing at the receiver. In such scenarios, low-precision phase configuration is sufficient to avoid that signals reflected by the individual elements are interfering destructively [28]. The power loss compared to a high-precision configuration is only a few $\mathrm{dB}[18]$. To limit the complexity of the prototype, we used two voltages to represent the phase states of $-\pi / 2$ and $\pi / 2$.

Based on the proposed element structure, we designed and fabricated an RIS board. It has the form of a Uniform Planar Array (UPA) with a $55 \times 20$ element grid. The front view of 
the RIS board is shown in Fig. 4. The size of the fabricated board is $80.08 \mathrm{~cm} \times 31.30 \mathrm{~cm}$, which also include the control circuit, i.e., the green part that does not have the capability of regulating the impinging radio waves. The size of the RIS part is $78.65 \mathrm{~cm} \times 20.54 \mathrm{~cm}$.

In the typical wireless applications, the users can move around freely in the azimuth plane, but do not have much freedom to move in the vertical direction. As a result, the RIS requires a high level of flexibility when controlling beam directions in the azimuth plane, while a lower accuracy is required in the vertical domain. We exploited this property to further simplify the implementation. More precisely, every five elements in each column form a group that shares the same bias voltage and, therefore, the same reflection coefficient. Hence, the total number of controllable bias signals is 220 . The control system is composed of an FPGA and 28 serial-in and parallel-out 8-bit shift registers, which generate parallel discrete bias voltages for the RIS elements. After that, the voltage is adjusted by a voltage level-shifter so that the phase difference at the working frequency of $5.8 \mathrm{GHz}$ is 180 degrees when the digital state changes from logic " 0 " to logic " 1 ". The bias voltages are applied to each element to adjust the junction capacitance of the varactor diodes.

\section{RIS-AIDED WIRELESS COMMUNICATION SYSTEM}

In this section, we describe the prototype of the RIS-aided wireless communication system shown in Fig. 5, which is composed of Personal Computers (PCs), universal software radio peripherals (USRPs), an RIS, an FPGA-based master control board, and the real-time RIS-UE feedback module.

The host computer, a USRP, and an antenna are the main components of the transmitter. The receiver consists of the same main components. These transceivers emulate an Access Point (AP) or base station (BS) and a user equipment (UE), respectively. The transmitter sends a video stream using a single passive antenna. The data is transmitted through the User Datagram Protocol (UDP). The receiver decodes the data stream and plays the video on the host computer.

Fig. 6 shows the hardware block diagram for the prototype of an RIS-aided wireless communication system. Table [III summarizes the details of the hardware modules. In the prototype system, the goal of the RIS is to serve as a smart reflector that "reflects" the impinging wireless signal as a beam towards the receiver. To support real-time adjustment of the reflection coefficients (e.g., to adapt the RIS to time-varying channel conditions or switch between serving different UEs), we introduce a feedback link [8] that connects the RIS board and the receiver with serial ports. The receiver feeds back the quality of the current channel (i.e., the received signal power), to the FPGA master control board. The feedback can be used to measure the effectiveness of the applied reflection coefficients. The next section will describe how we utilized this feedback for beamforming design.

The following part introduces the software framework of our communication system. The signal processing flow is shown in Fig. 7. First, our transmitter loads data provided by an external application, from a UDP socket. After the payload
TABLE III

DETAILED INFORMATION OF HARDWARE MOdUlES

\begin{tabular}{|c|c|}
\hline \hline Name & Description \\
\hline Central Controller & Computer with Intel Core i7-9700 processor \\
\hline SDR & NI USRP-2954R \\
\hline FPGA Module & Development board with Xilinx Zynq 7100 \\
\hline Antenna Gain & $17.1 \mathrm{dBi}$ gain @ 5.8 GHz \\
\hline Antenna Aperture & $169 \mathrm{~mm} \times 119 \mathrm{~mm}$ \\
\hline Antenna Beamwidth & $30^{\circ}$ \\
\hline \hline
\end{tabular}

data is encoded, the samples are mapped to the time-frequency resources. Afterwards, the Orthogonal Frequency Division Multiplexing (OFDM) modulation is applied, which converts the frequency-domain parallel signals to time-domain serial signals. The bandwidth and subcarrier spacing are $20 \mathrm{MHz}$ and $312.5 \mathrm{kHz}$, respectively, in our experiments. The signal is then upconverted to the carrier frequency and transmitted through the transmit antenna.

The receiver module performs the opposite functions as the transmitter. Synchronization and Carrier Frequency Offset (CFO) compensation are achieved by continuous measurement of both autocorrelations and cross correlations of the pilot signals. Note that the existing IEEE 802.11ac WiFi standards are adopted in this prototype system, and the over-the-air signaling of WiFi is kept intact. In other words, the integration of the RIS technology into the existing communication networks can be made seamless without modifications of the wireless communication protocols.

\section{RIS-AIDED BEAMFORMING METHOD}

The RIS will scatter or reflect the incident wave in a way determined by its configuration. In a scenario with a singleantenna transmitter and single-antenna receiver, as shown in Fig. 6, the communication performance is determined by the Signal-to-Noise Ratio (SNR). Hence, the RIS can aid the communication system by optimizing its reflection coefficients to maximize the received signal power. This effectively means that the RIS elements should make their individually reflected signals reach the receiver in phase (up to the granularity set by the hardware). If there is an LoS channel from the RIS to the receiver, the optimization will essentially result in a reflected beam in the angular direction leading to the receiver.

The RIS beamforming configuration problem has been analyzed mathematically in a series of previous works [29], [30]; we refer to [9] for a recent survey. In previous literature, the RIS configuration is generally divided into two disjoint subproblems: 1) selecting an SNR-maximizing configuration when having full Channel State Information (CSI) [10], [11]; 2) Acquiring CSI from explicit pilot signals [31]-[33]. The latter part is particularly challenging since the RIS (normally) does not have any RF chains, which prohibits it from directly obtaining CSI regarding the BS-RIS or RIS-UE channels using traditional pilot-based channel estimation methods [3]. Fulldimensional CSI acquisition requires the pilot signaling to grow linearly with the number of RIS elements [8]. To the best of our knowledge, the existing works rely on high-precision 


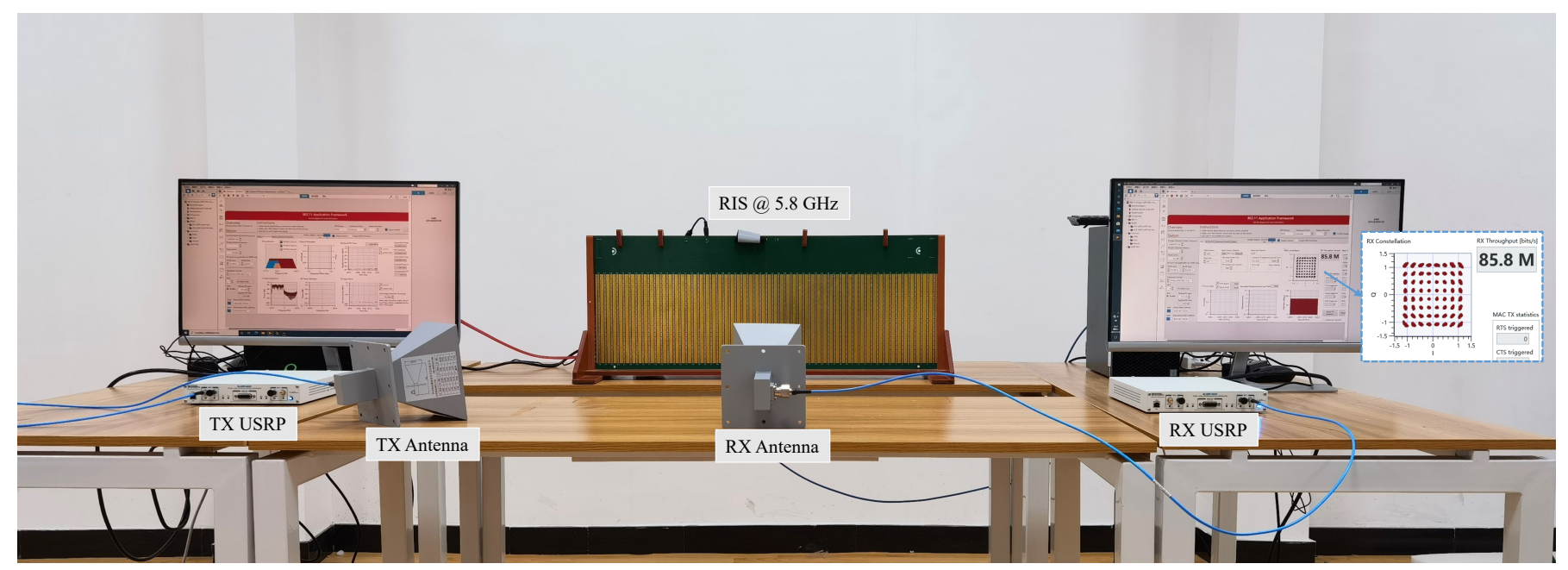

Fig. 5. The prototype of the RIS-aided wireless communication system.

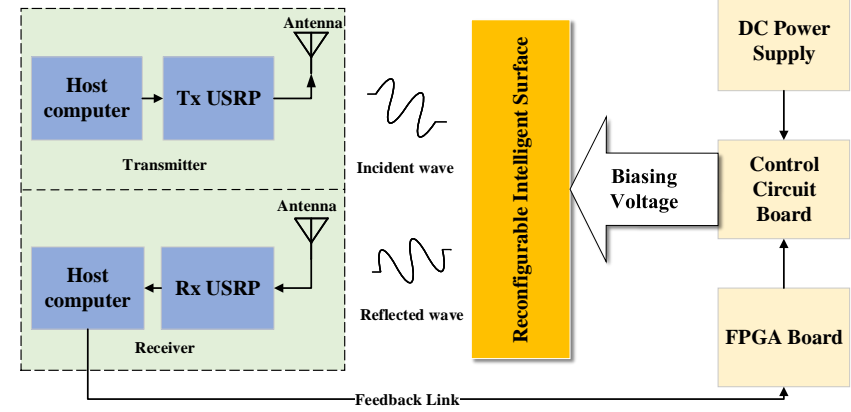

Fig. 6. System diagram for the RIS-aided communication system.

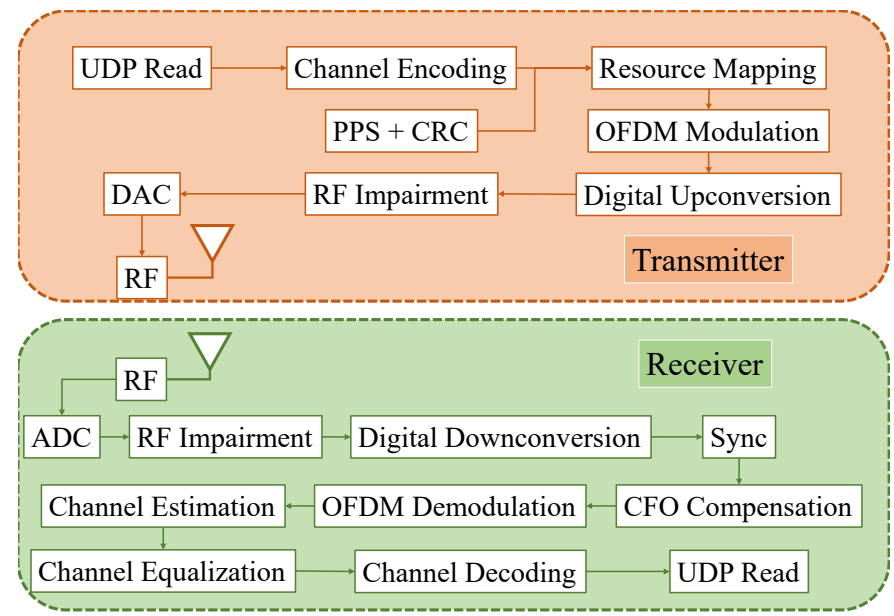

Fig. 7. The detail framework and the signal processing flow in USRPs.

configurations where an RIS with $L$ elements can switch between $L$ mutually orthogonal configurations. In summary, none of the existing algorithms was deemed to be implementable in our practical setup, where CSI acquisition and RIS configuration must be done jointly, and where there is only a low-bit resolution per element. Hence, we had to develop a new algorithm that could be utilized in the prototype system.

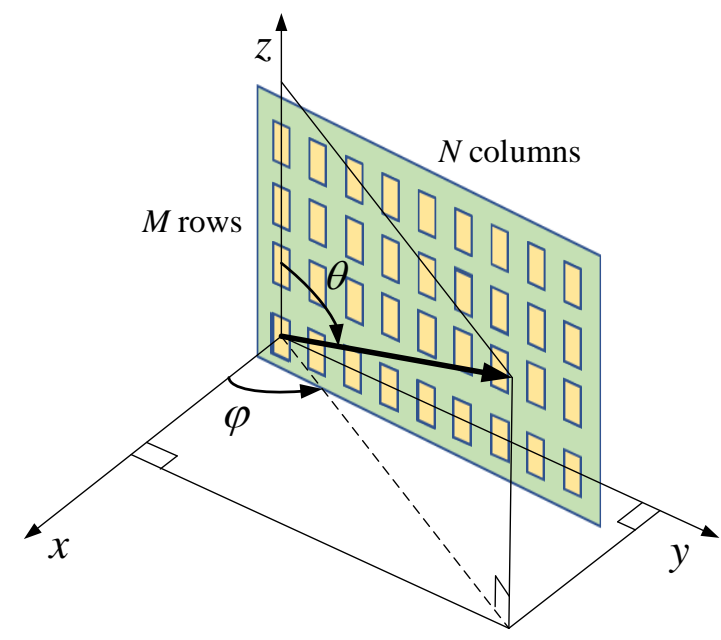

Fig. 8. The coordinate system describing the RIS with $M$ rows and $N$ columns.

\section{A. Codebook-based RIS Beamforming}

Since the fabricated RIS has the form of a UPA and is intended for scenarios with dominant LoS paths, we can exploit the geometry to develop a low-complexity method to configure the RIS. We will take inspiration from the codebook-based beamforming for UPAs. In traditional MIMO communications, a two-dimensional (2D) Discrete Fourier Transform (DFT) codebook can be used to specify a set of angular transmit beamforming directions when using a UPA. While in RISaided communications, the angle of the reflected beam depends on both the incident angle and the RIS configurations, we will show that for a given incident angle and a desired reflecting angle, the structure of the optimal RIS phase shifts is still in line with the 2D-DFT codebook.

As shown in Fig. 8, the RIS consists of a total of $L=M N$ elements, where $M$ represents the number of RIS elements in the $z$-axis direction (i.e., the number of rows), and $N$ represents the number of RIS elements in the $y$-axis direction (i.e., the number of columns). The elements are deployed on a rectangular grid with $d_{y}$ representing the spacing between 
the $y$-axis elements, and $d_{z}$ represents the spacing between the $z$-axis elements. As in most metamaterial designs, the vertical and horizontal spacings are smaller than half the wavelength, i.e., $d_{x} \leq \lambda / 2$ and $d_{y} \leq \lambda / 2$, where $\lambda$ is the wavelength of the intended signal.

The classical far-field approximation is known to be accurate even for large but finite surfaces [34] and will be utilized herein. The incident wave will consist of a superposition of multiple plane waves from different angles. We focus on one of them. The first reflecting element is at the origin. The zenith angle and azimuth angle to the transmitter, as seen from the origin, are $\theta$ and $\varphi$ respectively. For such an incident wave, the array response vector $\mathbf{a}(\theta, \varphi) \in \mathbb{C}^{L \times 1}$ of the UPA is [35], [36]:

$$
\mathbf{a}(\theta, \varphi)=\mathbf{a}_{y}(\theta, \varphi) \otimes \mathbf{a}_{z}(\theta),
$$

where $\otimes$ denotes the Kronecker product. $\mathbf{a}_{y}(\theta, \varphi)$ and $\mathbf{a}_{z}(\theta, \varphi)$ represent the array response vectors of a uniform linear array along the $y$-axis and the $z$-axis respectively:

$$
\begin{aligned}
& \mathbf{a}_{y}(\theta, \varphi)= \\
& \quad\left[1, e^{\frac{-j 2 \pi}{\lambda} d_{y} \sin \theta \sin \varphi}, \cdots, e^{\frac{-j 2 \pi}{\lambda} d_{y}(N-1) \sin \theta \sin \varphi}\right]^{\mathrm{T}}, \\
& \mathbf{a}_{z}(\theta)=\left[1, e^{\frac{-j 2 \pi}{\lambda} d_{z} \cdot \cos \theta}, \cdots, e^{\frac{-j 2 \pi}{\lambda} d_{z} \cdot(M-1) \cos \theta}\right]^{\mathrm{T}} .
\end{aligned}
$$

Note that in this model, we order the RIS elements column by column, starting from the origin 1 In the considered multicarrier system, the RIS-UE channel matrix is denoted as

$$
\mathbf{G}=\left[\mathbf{g}_{1}, \mathbf{g}_{2}, \cdots, \mathbf{g}_{K}\right] \in \mathbb{C}^{L \times K}
$$

where $K$ is the number of subcarriers and $\mathbf{g}_{k}$ is the channel vector at the $k$-th subcarrier, for $k=1, \ldots, K$. This channel matrix can be expressed as [35], [36]

$$
\mathbf{G}=\alpha_{g} \mathbf{a}\left(\theta_{g}, \varphi_{g}\right) \mathbf{b}^{\mathrm{T}}\left(\tau_{g}\right),
$$

where $\alpha_{g} \in \mathbb{C}$ is the complex path gain and $\mathbf{a}\left(\theta_{g}, \varphi_{g}\right)$ is the array response vector in (1) with $\theta_{g}$ and $\varphi_{g}$ being the zenith angle and azimuth angle of the UE. The delay response vector $\mathbf{b}\left(\tau_{g}\right) \in \mathbb{C}^{K \times 1}$ is defined as

$$
\mathbf{b}\left(\tau_{g}\right)=\left[\begin{array}{lll}
e^{-j 2 \pi f_{1} \tau_{g}}, & \cdots, & e^{-j 2 \pi f_{K} \tau_{g}}
\end{array}\right]^{\mathrm{T}},
$$

where $\tau_{g}$ is the path delay and $f_{k}$ is the frequency of the $k$-th subcarrier. If there are multiple paths, then $\mathbf{G}$ is computed as the summation over multiple terms of the type $\alpha_{g} \mathbf{a}\left(\theta_{g}, \varphi_{g}\right) \mathbf{b}^{\mathrm{T}}\left(\tau_{g}\right)$ with different values of $\alpha_{g}, \theta_{g}, \varphi_{g}$, and $\tau_{g}$ [36].

Similarly, the multi-carrier BS-RIS channel $\mathbf{H} \in \mathbb{C}^{L \times K}$ is denoted as

$$
\mathbf{H}=\left[\mathbf{h}_{1}, \mathbf{h}_{2}, \cdots, \mathbf{h}_{K}\right] \in \mathbb{C}^{L \times K},
$$

where $\mathbf{h}_{k}$ is the channel at the $k$-th subcarrier. This channel matrix can be computed as

$$
\mathbf{H}=\alpha_{h} \mathbf{a}\left(\theta_{h}, \varphi_{h}\right) \mathbf{b}^{\mathrm{T}}\left(\tau_{h}\right),
$$

\footnotetext{
${ }^{1}$ If they are ordered row by row, we simply need to switch the order of the Kronecker product in 1 .
}

where $\theta_{h}$ and $\varphi_{h}$ denote the zenith angle and azimuth angle of the BS, $\alpha_{h}$ is the complex path gain, and $\tau_{h}$ is the BSRIS path delay. If there are multiple paths, then (8) can be generalized as a summation over these terms.

In RIS-aided communications without a direct path, the received signal $y_{k} \in \mathbb{C}$ at the $k$-th subcarrier can be expressed as [12], [32]

$$
y_{k}=\left(\mathbf{g}_{k} \odot \mathbf{h}_{k}\right)^{\mathrm{T}} \boldsymbol{\omega} x_{k}+n_{k},
$$

where $x_{k} \in \mathbb{C}$ is the transmitted signal, $\odot$ denotes the Hadamard (element-wise) product, and $n_{k} \sim \mathcal{C N}\left(0, \sigma_{n}^{2}\right)$ is the white Gaussian noise with variance $\sigma_{n}^{2}$. The vector $\boldsymbol{\omega} \in \mathbb{C}^{L \times 1}$ contains the reflection coefficients of the RIS. The SNR at the $k$-th subcarrier is proportional to the end-to-end power gain $\left|\left(\mathbf{g}_{k} \odot \mathbf{h}_{k}\right)^{\mathrm{T}} \boldsymbol{\omega}\right|^{2}$ and can be tuned by the selection of $\boldsymbol{\omega}$.

The same $\boldsymbol{\omega}$ must be used on every subcarrier, which could lead to complicated RIS optimization problems [32]. We focus on scenarios with a single dominant path and utilize (5) and (8) to simplify the end-to-end power gain as

$$
\left|\left(\mathbf{g}_{k} \odot \mathbf{h}_{k}\right)^{\mathrm{T}} \boldsymbol{\omega}\right|^{2}=\left|\alpha_{g}\right|^{2}\left|\alpha_{h}\right|^{2}\left|\left(\mathbf{a}\left(\theta_{g}, \varphi_{g}\right) \odot \mathbf{a}\left(\theta_{h}, \varphi_{h}\right)\right)^{\mathrm{T}} \boldsymbol{\omega}\right|^{2},
$$

which is independent of the subcarrier index.

Since our reflecting elements are controlled by a single bias voltage, it does not have the freedom of independent phase and amplitude modifications. Therefore, we only consider the phase-shift functionality of each RIS element. If a continuous phase-shift optimization is possible, then the optimal phaseshift vector is given as [11], [32]

$$
\begin{aligned}
\boldsymbol{\omega}_{o} & =\underset{\left|[\boldsymbol{\omega}]_{i}\right|=1}{\arg \max }\left|\left(\mathbf{a}\left(\theta_{g}, \varphi_{g}\right) \odot \mathbf{a}\left(\theta_{h}, \varphi_{h}\right)\right)^{\mathrm{T}} \boldsymbol{\omega}\right|^{2} \\
& =\left(\mathbf{a}\left(\theta_{g}, \varphi_{g}\right) \odot \mathbf{a}\left(\theta_{h}, \varphi_{h}\right)\right)^{*},
\end{aligned}
$$

where $[\boldsymbol{\omega}]_{i}$ denotes the $i$-th entry of $\boldsymbol{\omega}$ and $*$ denotes the complex conjugate. The second equality follows from the Cauchy-Schwartz inequality. The optimal solution selects the phase-shifts so the reflected signals from all RIS elements arrive in phase at the receiver. By utilizing (1), we can rewrite (12) as

$$
\boldsymbol{\omega}_{o}=\left(\mathbf{a}^{(d)}\left(\theta_{g}, \varphi_{g}, \theta_{h}, \varphi_{h}\right)\right)^{*},
$$

where

$$
\begin{aligned}
& \mathbf{a}^{(d)}\left(\theta_{g}, \varphi_{g}, \theta_{h}, \varphi_{h}\right)= \\
& \mathbf{a}_{y}^{(d)}\left(\theta_{g}, \varphi_{g}, \theta_{h}, \varphi_{h}\right) \otimes \mathbf{a}_{z}^{(d)}\left(\theta_{g}, \theta_{h}\right)
\end{aligned}
$$

with

$$
\begin{gathered}
\mathbf{a}_{y}^{(d)}\left(\theta_{g}, \varphi_{g}, \theta_{h}, \varphi_{h}\right)=\left[1, e^{\frac{-j 2 \pi}{\lambda} d_{y}\left(\sin \theta_{h} \sin \varphi_{h}+\sin \theta_{g} \sin \varphi_{g}\right)},\right. \\
\ldots, e^{\left.\frac{-j 2 \pi}{\lambda} d_{y}(N-1)\left(\sin \theta_{h} \sin \varphi_{h}+\sin \theta_{g} \sin \varphi_{g}\right)\right]^{\mathrm{T}}} \\
\mathbf{a}_{z}^{(d)}\left(\theta_{g}, \theta_{h}\right)=\left[1, e^{\frac{-j 2 \pi}{\lambda} d_{z}\left(\cos \theta_{h}+\cos \theta_{g}\right)}\right. \\
\left.\cdots, e^{\frac{-j 2 \pi}{\lambda} d_{z}(M-1)\left(\cos \theta_{h}+\cos \theta_{g}\right)}\right]^{\mathrm{T}}
\end{gathered}
$$

The solution in (14) has a structure that can be exploited in calculating the reflection coefficients. Note that both $\mathbf{a}_{y}^{(d)}\left(\theta_{g}, \varphi_{g}, \theta_{h}, \varphi_{h}\right)$ and $\mathbf{a}_{z}^{(d)}\left(\theta_{g}, \theta_{h}\right)$ have the structure 
$\left[1, \xi, \xi^{2}, \ldots\right]^{\mathrm{T}}$, but with different complex exponentials $\xi$ and lengths. With the increase in the number of elements, such vectors can be well approximated by the columns of the DFT matrix [37], [38]. We denote the DFT matrix of size $K \times K$ as

$$
\mathcal{F}(K)=\left[\begin{array}{ccccc}
1 & 1 & 1 & \cdots & 1 \\
1 & \xi & \xi^{2} & \cdots & \xi^{K-1} \\
1 & \xi^{2} & \xi^{2 \cdot 2} & \cdots & \xi^{2(K-1)} \\
\vdots & \vdots & \vdots & \ddots & \vdots \\
1 & \xi^{K-1} & \xi^{2(K-1)} & \cdots & \xi^{(K-1)(K-1)}
\end{array}\right],
$$

where $\xi=e^{-j(2 \pi / K)}$.

For the considered UPA architecture, we can build a beamforming codebook for the RIS as $\mathcal{F}(M) \otimes \mathcal{F}(N)$. Therefore, each column of $\mathcal{F}(M) \otimes \mathcal{F}(N)$ forms a codeword representing the reflection of an incident beam in a certain beam direction. When $M$ and $N$ are large, the codewords will closely approximate all RIS configurations for which an incident plane wave is beamformed in a distinct angular direction.

In our prototype, the phase shifts are quantized to being either $-\pi / 2$ or $+\pi / 2$, thus the above-described solution cannot be utilized. Hence, the corresponding problem is

$$
\begin{aligned}
\hat{\boldsymbol{\omega}}_{o} & =\underset{[\boldsymbol{\omega}]_{i} \in\left\{e^{-j \pi / 2}, e^{j \pi / 2}\right\}}{\arg \max }\left|\left(\mathbf{a}\left(\theta_{g}, \varphi_{g}\right) \odot \mathbf{a}\left(\theta_{h}, \varphi_{h}\right)\right)^{\mathrm{T}} \boldsymbol{\omega}\right|^{2} \\
& =\underset{[\boldsymbol{\omega}]_{i} \in\left\{e^{-j \pi / 2}, e^{j \pi / 2}\right\}}{\arg \max }\left|\boldsymbol{\omega}_{o}^{\mathrm{H}} \boldsymbol{\omega}\right|^{2},
\end{aligned}
$$

that finds the quantized vector that gives the maximum inner product with the ideal solution in (12). One solution is obtained by making sure all terms in the inner product in (19) have a positive real part:

$$
\left[\hat{\boldsymbol{\omega}}_{o}\right]_{i}= \begin{cases}e^{j \pi / 2}, & \text { if } \arg \left(\left[\boldsymbol{\omega}_{o}\right]_{i}\right) \in[-\pi, 0) \\ e^{-j \pi / 2}, & \text { if } \arg \left(\left[\boldsymbol{\omega}_{o}\right]_{i}\right) \in[0, \pi) .\end{cases}
$$

In practice, when the angular information is not directly available, one may search through the codebook and find the codeword that gives the maximum received power. Although the codebook is designed based on far-field array response vectors, only a minor loss in beamforming gain is expected when operating the system at distances shorter than the Fraunhofer distance. We recall from [34] that the main near-field effects only occurs when the propagation distance is proportional to the RIS size (in $80 \mathrm{~cm}$ in our prototype).

\section{B. Proposed Fast Beamforming Algorithm}

Most previous algorithms for channel estimation and RIS configuration treat these problems separately, consider arbitrary channel structure, and assume infinite phase-shift precision. However, recent works have shown that the most compelling advantage of the RIS technology exists when the transmitter and receiver have a single dominant path to the RIS, and the RIS is configured based on these paths [12], [32]. This insight is utilized in our prototype to greatly reduce the training/feedback overhead when configuring the surface. We will now describe the proposed RIS beamforming algorithm, which will be evaluated experimentally.

When there are dominant angular paths, the desired configuration will be of the form in (13), which is approximately a column of $\mathcal{F}(M) \otimes \mathcal{F}(N)$. When quantizing the elements of the codewords to be either $e^{-j \pi / 2}$ or $e^{j \pi / 2}$, many adjacent elements will have the same phase shift. We will utilize this property to develop an algorithm that gradually changes the phase shifts on a column-by-column or row-by-row basis to gradually increase the SNR of the end-to-end link.

The basic idea of our proposed greedy algorithm is to invert the phases of a certain row or column of the RIS. If this configuration state increases the power of the received signal, compared to the previous state, the new state is utilized. The algorithm is enabled by our UE-RIS feedback module. The details are given in Algorithm [1. Note that the reflection coefficients at time index $t$ are now denoted by an $M \times N$ complex matrix $\mathbf{R}_{t}$ for ease of exposition. The vector $\boldsymbol{\omega}$ is the vectorization of this matrix. The $(m, n)$-th element in $\mathbf{R}_{t}$ is the reflection coefficient of the element located at the $m$-th row and $n$-th column of the RIS. $\mathbf{R}_{t}$ can also be written as

$$
\mathbf{R}_{t}=\left[\mathbf{r}_{t, 1}, \mathbf{r}_{t, 2}, \cdots, \mathbf{r}_{t, N}\right]=\left[\mathbf{s}_{t, 1}, \mathbf{s}_{t, 2}, \cdots, \mathbf{s}_{t, M}\right]^{\mathrm{T}},
$$

where $\mathbf{r}_{t, n} \in \mathbb{C}^{M \times 1}, n=1, \ldots, N$ are the coefficients of the $n$-th column and $\mathbf{s}_{t, m} \in \mathbb{C}^{N \times 1}, m=1, \ldots, M$ are the coefficients of the $m$-th row. We can switch the states of a column by changing its sign, since $e^{-j \pi / 2}=-j$ and $e^{j \pi / 2}=j$. We denote the average power of the received signal at time index $t$ as $p_{t}$, which is computed over the whole signal bandwidth in order to reduce the influence of noise.

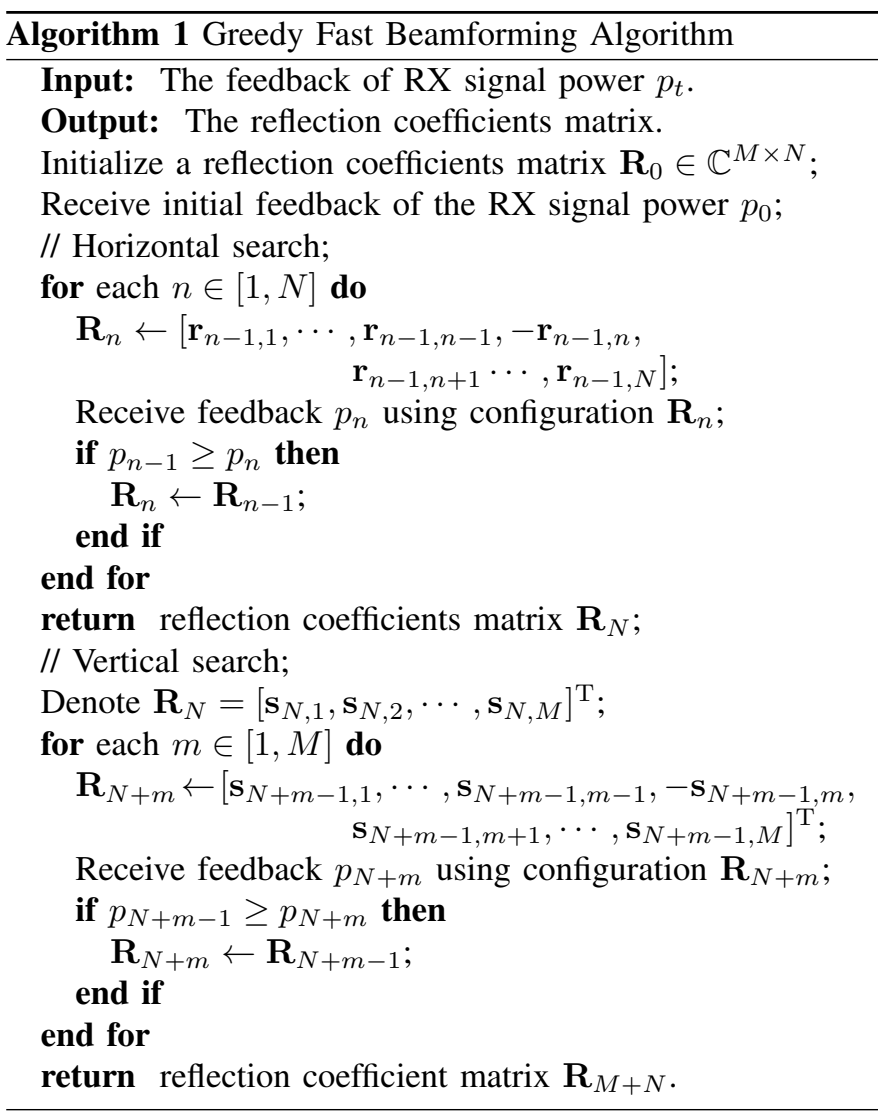

Algorithm 11 determines the reflection coefficients using $M+N$ feedback iterations, which is much smaller than the $M N$ iterations required for estimating unstructured channels [8]. The computational complexity order is $\mathcal{O}(M+N)$. In 
addition, it does not introduce significant fluctuations of the received power, thus data transmission can be carried out simultaneously. The received power can be measured by the wideband SNR or Reference Signal Receiving Power (RSRP), or other similar metrics that reflect the channel quality. In particular, existing RSRP measurements can be utilized so that extra signaling is not needed. It is also possible to repeat Algorithm 11 several times to refine the coefficients, and even keep it iterating during data transmission to track the UE's movement. We have observed from our experiments that ten iterations of Algorithm 1 brings around $1 \mathrm{~dB}$ gain compared to the case of single iteration. The algorithm can be initialized in different ways. We propose to start from a homogeneous configuration (i.e., all elements have the same state), which corresponds to the behavior of a conventional homogeneous reflective surface with the same absorption properties. In this way, the algorithm is guaranteed to outperform such a surface.

Even if the algorithm was designed particularly for scenarios with one dominant far-field path to the RIS and one dominant far-field path from the RIS, it can be applied under any circumstances (e.g., in near-field propagation) and will monotonically increase the SNR, thus it is also guaranteed to converge (if the channel conditions are static). In the next section, we show the effectiveness of the proposed algorithm through experiments.

\section{EXPERIMENTAL RESULTS}

In this section, we present the results from several experiments that were made using our prototype to verify the performance of this proof-of-concept. First, we test the electromagnetic reflection characteristics of the RIS. Next, we verify the channel reciprocity, which is of particular value for communication theorists and engineers. We then test the radiation pattern in an anechoic chamber to measure the beamforming function of our RIS. Finally, we present and analyze the results from indoor and outdoor field trials.

\section{A. Bias Voltage Response Test}

Due to the tolerances of the PCB manufacturing process, the difference in the dielectric constant of the material, and the non-ideal characteristics of the varactor diode, the electromagnetic characteristics of a fabricated RIS are often different from the ideal simulated one [25]. To achieve precise control of the reflected signal, we measure the relationship between the reflection coefficients and the bias voltage of the varactor diode. We use horn antennas to transmit and receive signals that are reflected by the RIS. Two antennas are placed $1 \mathrm{~m}$ away from the metasurface, and a sinusoidal signal at 5.8 $\mathrm{GHz}$ is used for the excitation. As shown in Fig. 9, when the bias voltage gradually increases from 0 to $16 \mathrm{~V}$, the experimental results show that the gain fluctuation is $6.5 \mathrm{~dB}$ and the range of phase shifts is 250 degrees. Note that the phases are normalized such that the phase shift is zero when the bias voltage is $0 \mathrm{~V}$. The gain is also normalized and the maximum value is $0 \mathrm{~dB}$. We observe from Fig. 9 that the reflection behavior is well in line with the CST simulation results. However, the control voltages to generate each states of

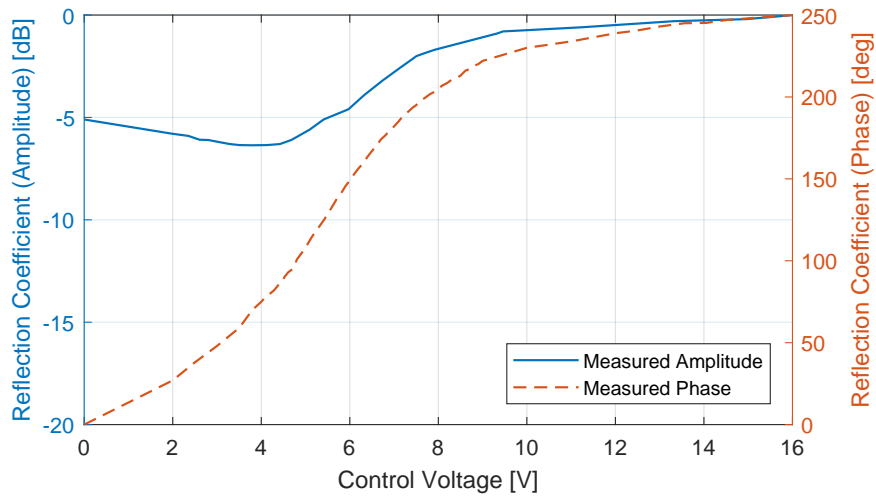

Fig. 9. Measured relationship between the control voltage of RIS and the gain and phase of the reflection coefficient at $5.8 \mathrm{GHz}$. The angles of incidence and reflection are both $45^{\circ}$.

phase shift are not identical to the simulated ones. In order to achieve the desired reflection coefficients, the control voltages need to be selected according to measurements. Hence, when designing a 1-bit RIS where each element can take two states having 180 degree phase difference, measurements must be made to identify the right bias voltages. We find that when the bias voltages are $3.8 \mathrm{~V}$ and $16 \mathrm{~V}$, the phase shifts are 70 degrees and 250 degrees, respectively, which leads to the desired phase difference of 180 degrees.

We tested the voltage-phase response at different incident angles: $15^{\circ}, 30^{\circ}$, and $45^{\circ}$ in the azimuth plane. The results are shown in Fig. 10 When the bias voltage increases from 0 to $16 \mathrm{~V}$, a dynamic phase range of about $276^{\circ}$ can be achieved at $15^{\circ}, 265^{\circ}$ can be achieved at $30^{\circ}$, and only $250^{\circ}$ can be achieved at $45^{\circ}$. Importantly, the experimental results show that the response of the RIS elements is angle-dependent, thus one may not find a pair of bias voltages that will result in exactly 180 degrees phase difference for any incident angles. A larger incident angle results in smaller phase variations. This observation is in line with the results in [39], where the phase variations due to different incident angles were even bigger. The likely physical explanation of this phenomenon is that the reflectance of the element depends on the incident angle [40]. This property has not been taken into consideration in the canonical system model used by the communication and signal processing literature [9], [12]. It is important to evolve the models since angle-dependence implies that different paths in a multipath scenario will be subject to different phase shifts, and that one cannot design a codebook of RIS configuration vectors that are orthogonal for all incident angles.

\section{B. Channel Reciprocity Test}

The time division duplex (TDD) mode is widely adopted in $5 \mathrm{G}$ mobile communication systems and $\mathrm{WiFi}$. One feature is that the uplink (UL) and downlink (DL) channels are reciprocal, which is a great advantage when having many antennas on one side of the link, because one can then learn the channels in the direction where the channel estimation requires the least signaling. For example, to support an arbitrarily large number of base station antennas, massive MIMO builds on the 


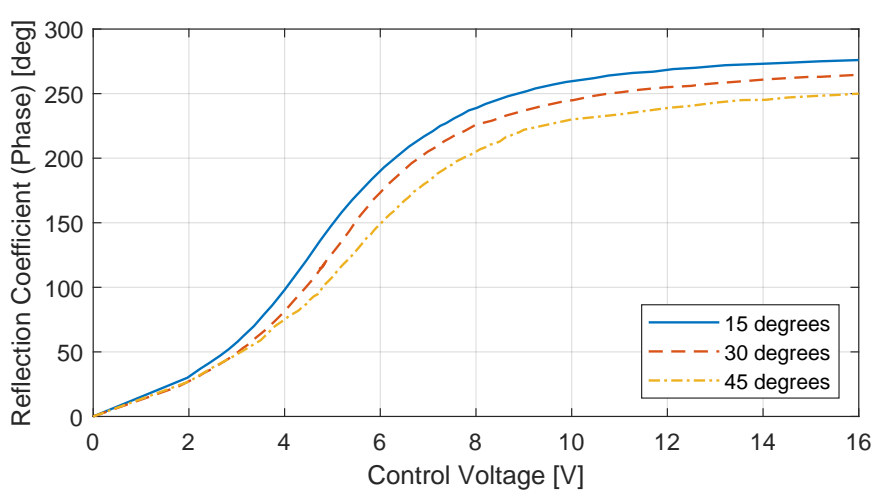

Fig. 10. Measured relationship between the control voltage of the RIS and the phase of reflection coefficients with different incident angles at $5.8 \mathrm{GHz}$.

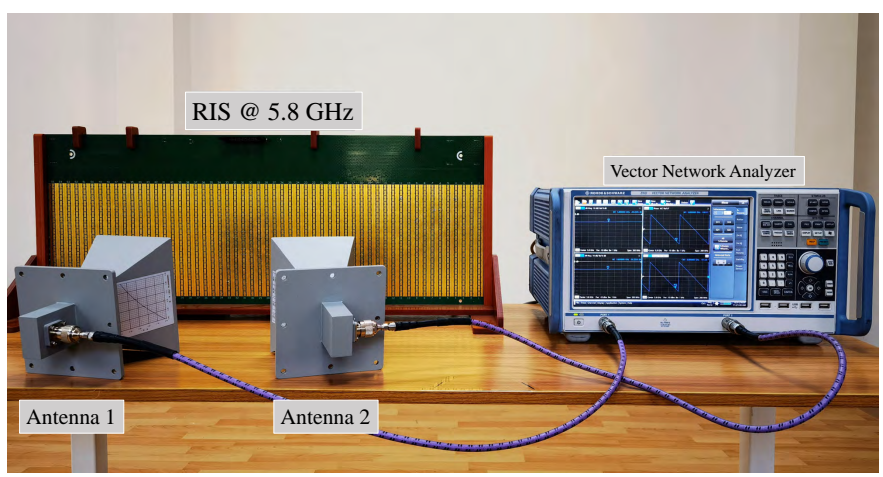

Fig. 11. Channel reciprocity and reflection coefficient test environment.

principle of estimating channels in the UL and then uses the estimates also for DL precoding [3].

When it comes to RIS-aided communications, UL/DL channel reciprocity would imply that the RIS can be configured to beamforming signals in UL and then work equally well in the DL without having to be reconfigured. Motivated by this, we conduct experiments to verify the UL/DL channel reciprocity of RIS. We use the vector network analyzer to test the S-parameters of the UL and DL. The test environment is shown in Fig. 11, and two horn antennas are placed close in front of the RIS to minimize the effects of multipaths other than the one reflected by the RIS. A lot of other trials have been made with different configurations and antenna positions. However since they lead to the same conclusion, we only show one example of the measured S12 and S21 parameters of the RIS wireless channel in Fig. 12. It can be seen from the figure that in the amplitude-frequency curve and phase-frequency curve, the S12 and S21 parameters are quite consistent with each other. Hence, despite the incidentangle-dependent behavior, the UL and DL channels in RISaided communication are reciprocal in our case. It means a channel estimate computed in one TX-RX direction can be reused when transmitting in the opposite direction, provided that the configuration remains unchanged. It also implies that if we configure the RIS for one direction, then we may keep the configuration when transmitting in the opposite direction.

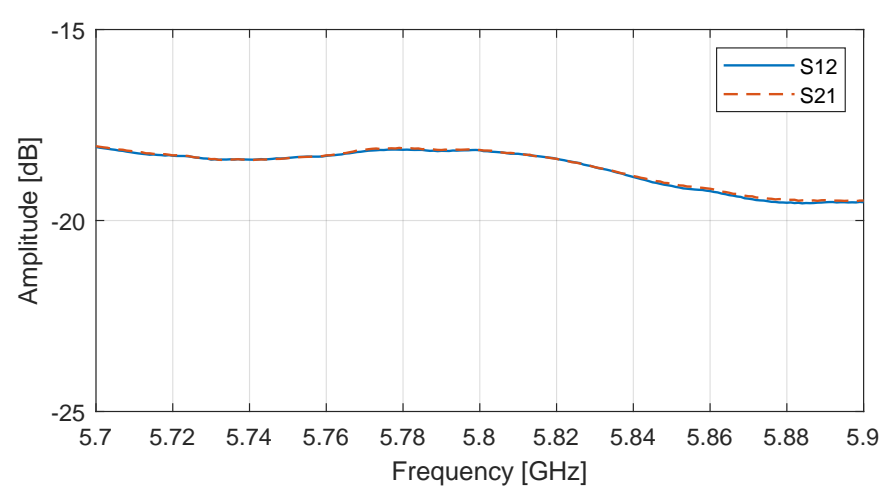

(a) Amplitude vs. frequency

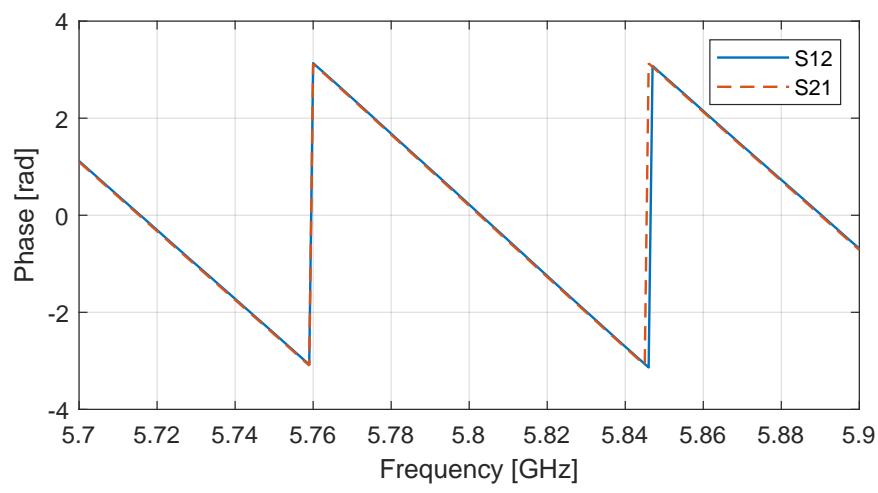

(b) Phase vs. frequency

Fig. 12. Measured S12 and S21 parameters of the RIS wireless channel, frequency range from $5.7 \mathrm{GHz}$ to $5.9 \mathrm{GHz}$.

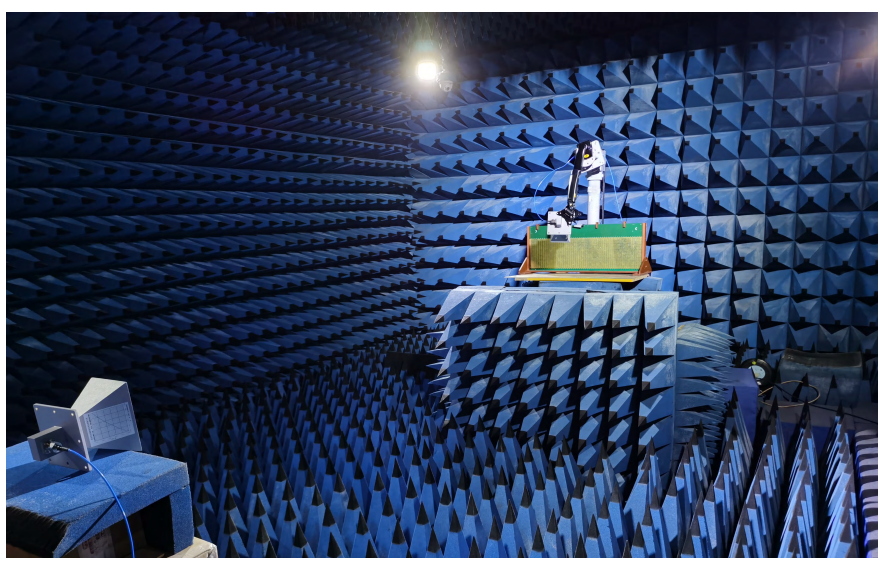

Fig. 13. The microwave anechoic chamber of size $4 \mathrm{~m} \times 6 \mathrm{~m} \times 4 \mathrm{~m}$. The RIS-RX antenna distance is $4.8 \mathrm{~m}$. The TX-RIS distance is $40 \mathrm{~cm}$.

\section{Radiation Pattern Test}

Next, we test the beamforming performance of the fabricated RIS. The experiment is carried out in a microwave anechoic chamber as shown in Fig. 13. The RIS and transmit antenna are fixed on a rotating platform, with the transmit antenna facing the RIS.

We adjusted the reflection coefficients according to the 2DDFT codebook described in Section IV to form a beam in the azimuth direction of 30 degrees. The codewords were 1-bit quantized, as previously described. The platform was rotated to measure the radiation pattern. The result is shown in Fig. 14 


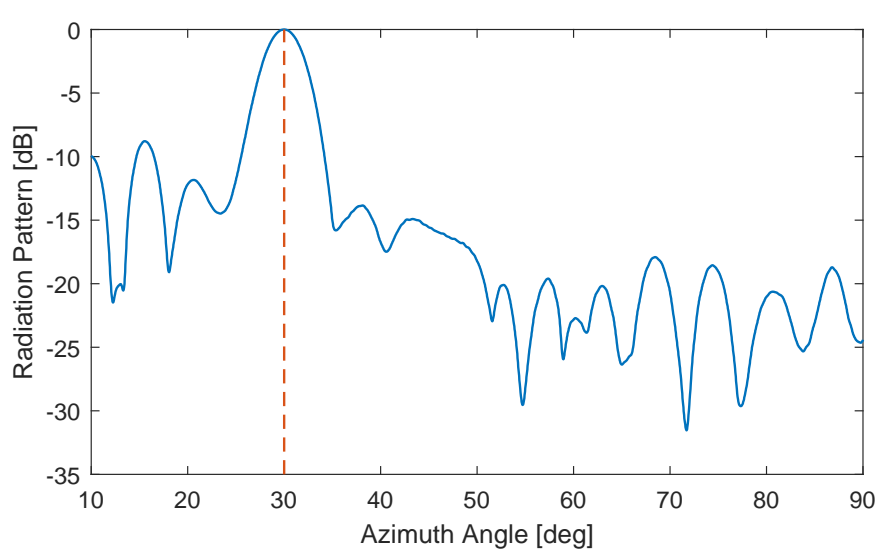

Fig. 14. The measured radiation pattern of the RIS with pre-defined reflection coefficients.

where the gains are normalized so the maximum value is $0 \mathrm{~dB}$. The measurement shows that a high gain beam is generated in a direction of $30^{\circ}$ as expected. The half-power beamwidth is $5.2^{\circ}$. The largest side lobes is $-8.79 \mathrm{~dB}$ and is located to the left, while the largest sidelobe at the right side is -11.8 $\mathrm{dB}$.

\section{Indoor Over-the-air Test}

We will now present results from the over-the-air test that was made in the indoor environment shown in Fig. 15 (a). The transmitter is placed in the corridor and the receiver is in a room next to it. The transmitter and receiver are separated by a $30 \mathrm{~cm}$ thick concrete wall, which includes a $53 \mathrm{~cm}$ thick pillar in the middle. In such a case without an LoS path, the transmitted signal suffers from penetration loss before reaching the RIS. Nevertheless, the proposed Algorithm 1 can be utilized to configure the RIS to gradually increase the received signal power. Note that the RX received power is utilized during the feedback. It is not perfect due to the influence of noise, interference, and time-varying channel. However, we may average the received power over time in order to make the algorithm more stable. Fig. 15.(b) shows the received baseband power when using the RIS (after Algorithm 1 has finished). Fig. 15 (c) shows the corresponding received power in the reference case where the RIS is replaced with a copper plate (shown in Fig. 16 (b)). Note that the position and angle of the copper plate are the same as the RIS. The curve marked with "packet start" is the received power when a packet is successfully decoded, while the "current" curve is the power at current moment, which may not have data transmission. The results show that an RIS configured using the proposed algorithm brings a power gain of around $26 \mathrm{~dB}$. While it is generally recognized that the RIS technology is effective in LoS scenarios [12], our experiment shows that an RIS can be very effective also in non-LoS scenarios, at least over short distances.

\section{E. Outdoor Over-the-air Test}

We will now describe our outdoor field trials. The testing sites were chosen at the campus of Huazhong University

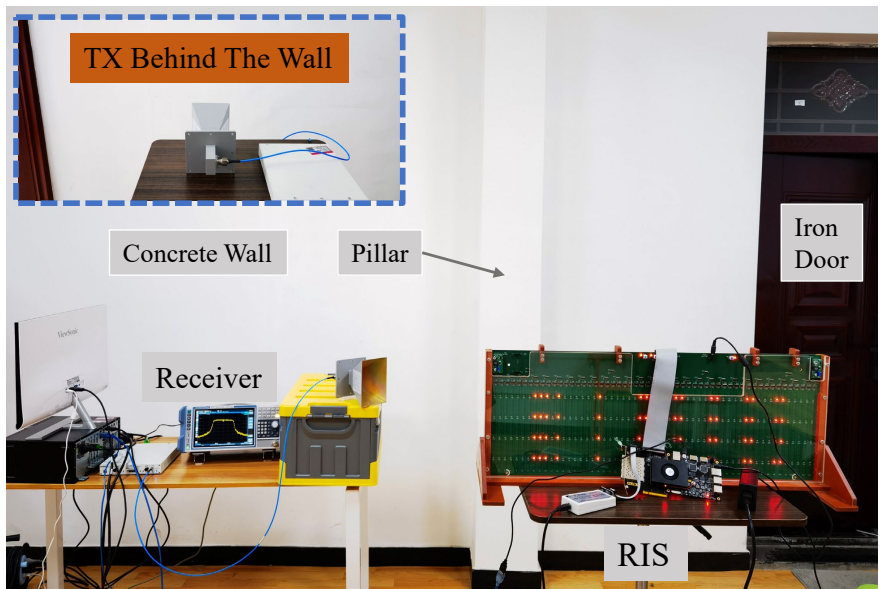

(a)

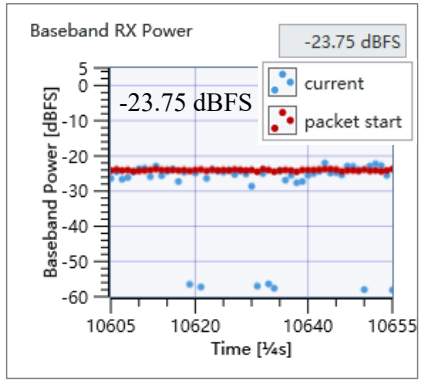

(b)

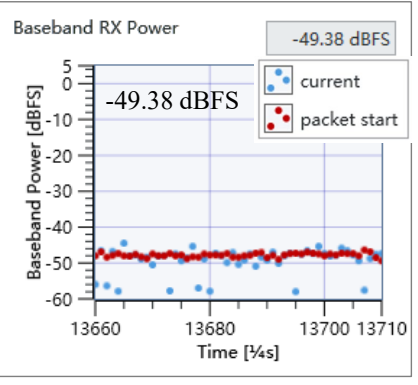

(c)
Fig. 15. Indoor non-LoS test: (a) the testing scenario: the transmitter is behind a $30 \mathrm{~cm}$ concrete wall pointing at the RIS, the the thickness of reinforced concrete pillar is $53 \mathrm{~cm}$, the iron door is closed, and the transmit power is $-16 \mathrm{dBm}$; (b) the Baseband RX power is around $-23.75 \mathrm{dBFS}$ when using RIS; (c) the Baseband RX power is -49.38 dBFS when using a copper plate.

TABLE IV

SySTEM PARAMETERS OF OUTDOOR TEST

\begin{tabular}{|c|c|}
\hline Parameter & Value \\
\hline Center frequency & $5.8 \mathrm{GHz}$ \\
\hline Modulation method & OFDM \\
\hline Bandwidth & $20 \mathrm{MHz}$ \\
\hline TX-RX distance & $50 \mathrm{~m}$ or $500 \mathrm{~m}$ \\
\hline Transmit power & $13 \mathrm{dBm}$ or $23 \mathrm{dBm}$ \\
\hline
\end{tabular}

of Science and Technology (HUST). Table IV summarizes the main parameters of the system settings. We chose two propagating distances for the field trial: $50 \mathrm{~m}$ and $500 \mathrm{~m}$.

1) 50 meter test: Fig. 16 (a) shows the environment, including the transmitter, receiver, and RIS locations, in the $50 \mathrm{~m}$ experiment. This field trial was carried out on the roof of our laboratory. The transmit power was $13 \mathrm{dBm}$. Fig. 16 (c) and Fig. 16 (d) show the spectrum of the receiver with and without RIS, respectively. The spectrum in Fig. 16 (c) was measured at the receiver side using a spectrum analyzer in the case that the RIS had been configured using our proposed Algorithm 1] Fig. 16 (d) shows the spectrum when the RIS is replaced with a copper plate as shown in Fig. 16 (b). The results in Fig. 16 (c) and Fig. 16 (d) show a $27 \mathrm{~dB}$ power gain when using the RIS, as compared to using the copper plate. This number is well aligned with the power gain observed in 
the indoor test.

It is generally hard to compare measurement results like this with theory since there are many sources of uncertainty. However, to put the numbers into perspective, suppose the copper plate would behave similarly to an RIS that has a random configuration. In that case, a fully optimized RIS beamforming could provide an average power gain up to $1100=30.4 \mathrm{~dB}$. Furthermore, it is known that 1-bit RIS configurations suffer a $-3.9 \mathrm{~dB}$ loss on average [18]. Hence, the predicted power gain in this setup is $26.5 \mathrm{~dB}$, which is very much aligned with the measurement results. While this is not an exact calculation, it gives a first-order indication that the RIS prototype with the proposed algorithm performs according to theory.

2) 500 meter test: Fig. 17 shows the environment of our $500 \mathrm{~m}$ field trial, which we believe is the longest distance that has been considered so far in the literature on RIS-aided communications. The experiment was carried out between two buildings. The transmit power was $23 \mathrm{dBm}$. Due to the long distance and limited transmit power, high-order modulation was not supported. The maximum transmission rate in the measurement was $32.1 \mathrm{Mbps}$, which was achieved using 20 $\mathrm{MHz}$ of bandwidth and 16 QAM modulation. Fig. 18 shows the spectrum of the received signal using the RIS (with the proposed algorithm) and using the copper plate. In this experiment, the power gain is $14 \mathrm{~dB}$, which was sufficient to enable real-time transmission of a video with $1920 \times 1080$ resolution. The video was only playing smoothly when using the RIS.

The measured power gain is smaller than in the shortdistance experiments. There are several possible explanations for this result. In general, the total received power originates both from the path via the RIS (or copper plate) and the combination of the multi-paths that are not involving the RIS. One possible explanation is that the RIS provides the same improvements over the copper plate as before, but the impact on the total received power is smaller since the longer distance makes the path via the RIS proportionally weaker compared to the surrounding multi-paths. Another possibility is that the more complicated propagation environment makes the channels via the RIS frequency-selective, which effectively reduces the beamforming gain since no RIS configuration fits for the entire band.

Note that the gain of the RIS depends on multiple parameters [41], which includes the field pattern of the RIS elements, the distances between the RIS and the antennas, the angles of incidence/reflection, the number of the reflection elements, etc. A more detailed study is provided in [42].

\section{F. Power Consumption}

Finally, we provide a detailed description of the power consumption of the fabricated RIS. The power consumed by the individual electronic components on the RIS board is shown in Table $\mathrm{V}$

It is interesting to note that the varactor diodes consume little power, although they are large in number. This is because reverse bias voltages are applied to them. Most of

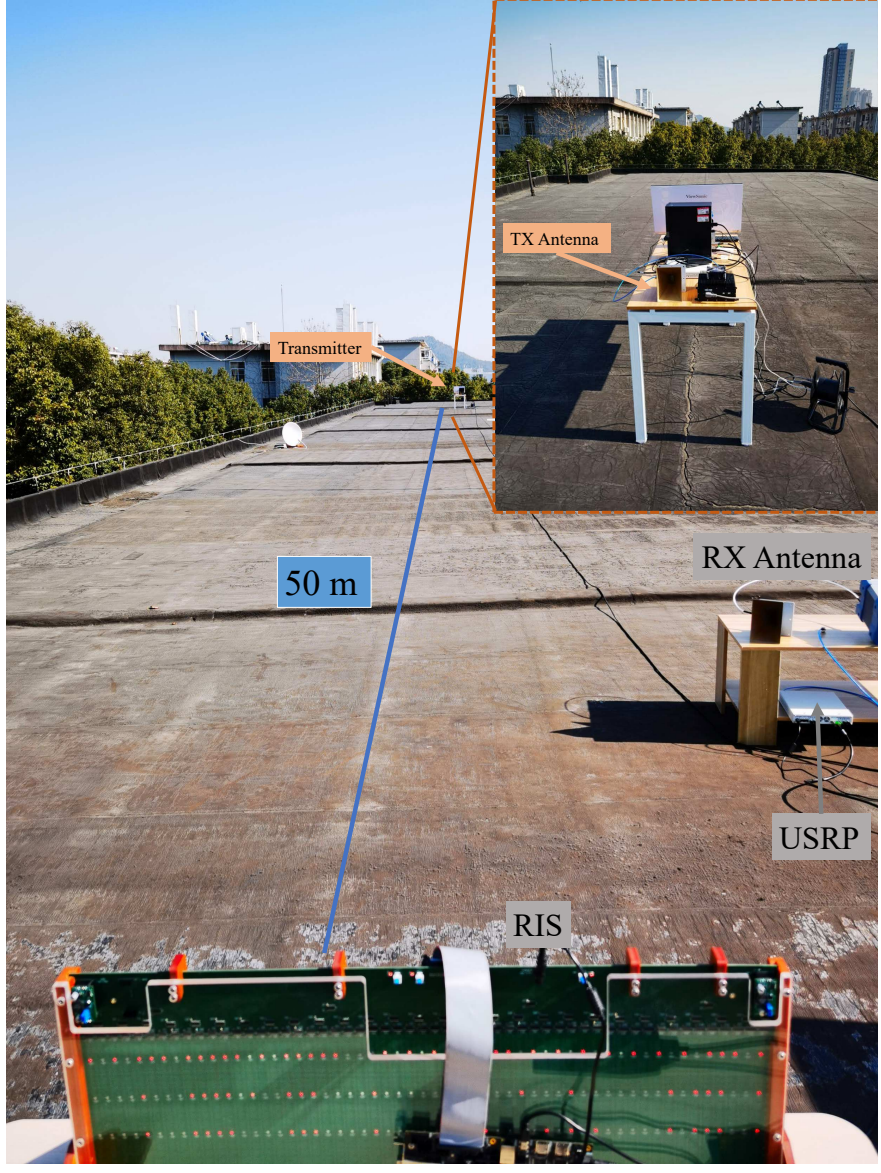

(a)

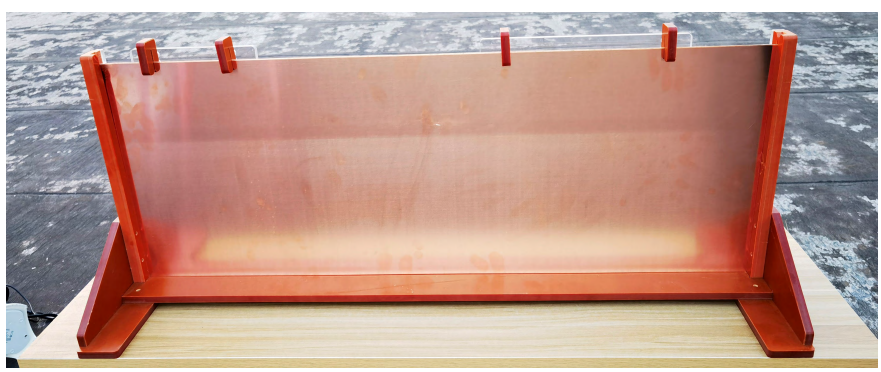

(b)

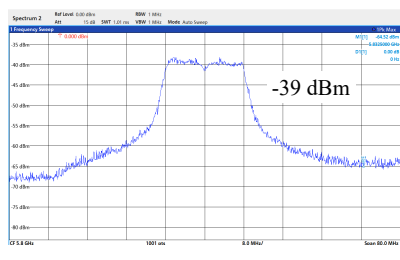

(c)

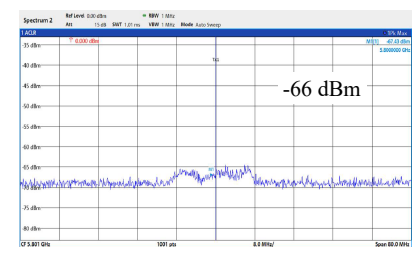

(d)
Fig. 16. The $50 \mathrm{~m}$ outdoor over-the-air test: (a) the scene of the transmitter and receiver; (b) a copper plate of the same size as the RIS; (c) spectrum when using the RIS; (d) spectrum when using the copper plate.

the power is spent on the chips of the level regulators, to enable continuous bias voltage adjustment. This is useful for experiments. However, in a commercial product, it may not be necessary and low-power alternatives are available. Apart from the RIS board, the high-end FPGA controller consumes $1.5 \mathrm{~W}$ of power in our prototype. Nevertheless, the proposed 


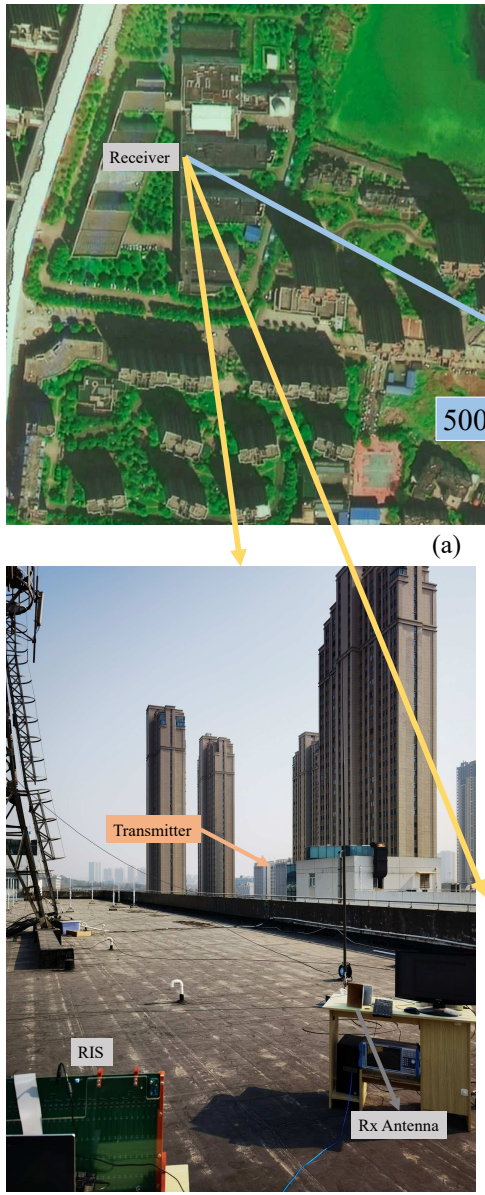

(c)

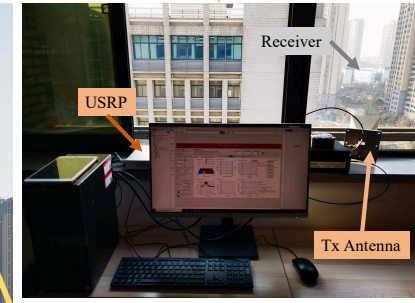

(b)

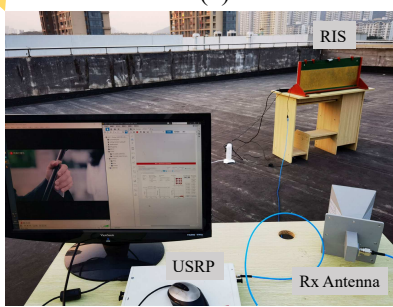

(d)
Fig. 17. The $500 \mathrm{~m}$ outdoor over-the-air test: (a) location of the transmitter and receiver on the map; (b) the transmitter; (c) the site of the receiver; (d) a photo of the receiver when playing a live video.

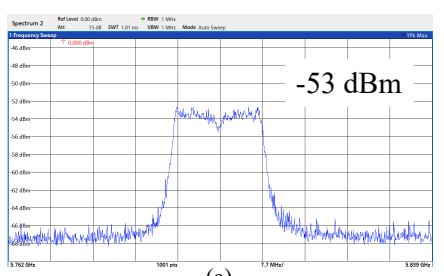

(a)

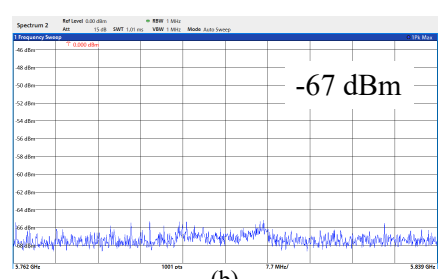

(b)
Fig. 18. The spectrum of $500 \mathrm{~m}$ outdoor over-the-air test: (a) using the RIS; (b) using the copper plate.

TABLE V

POWER CONSUMPTION BREAKDOWN FOR RIS BOARD

\begin{tabular}{|c|c|}
\hline Name & Power \\
\hline Bidirectional voltage translators & $0.0138 \mathrm{~W}$ \\
\hline Level regulators & $0.918 \mathrm{~W}$ \\
\hline Varactor diode & $0.00176 \mathrm{~W}$ \\
\hline Total & $\mathbf{0 . 9 3 4} \mathbf{W}$ \\
\hline
\end{tabular}

beamforming algorithm could also be implemented on an ordinary microcontroller. This would bring down the power consumption of the controller to around $10 \mathrm{~mW}$. In summary, we predict that an equal-sized RIS designed for minimum power consumption could consume far below $1 \mathrm{~W}$.

\section{CONCLUSION}

In this paper, we described our design and fabrication of an RIS with 1100 controllable elements working in the 5.8 $\mathrm{GHz}$ band. It was used to prototype an RIS-aided wireless communication system, where each element has two states (1bit RIS). We proposed a self-adaptive RIS beamforming configuration algorithm that is based on real-time feedback with the help of a RIS-UE link. The geometrical channel properties and 1-bit structure were exploited to minimize the number of feedback iterations. The algorithm is particularly suited for setups where there are LoS paths from and to the RIS (e.g., where the RIS has been deployed to overcome shadowing), and can be utilized in any frequency band, from sub-6 GHz to sub-terahertz. The proposed algorithm can be added on top of existing wireless protocols since only standard received power measurements are utilized when selecting the configuration. We first evaluated basic propagation properties in an anechoic chamber. We noticed that the reflection coefficients of the RIS elements are varying with the incident angle, verified that channel reciprocity holds, and demonstrated the effectiveness of the reconfigurable beam-steering capability by measuring the radiation pattern.

We also evaluated the prototype in indoor and outdoor field trials. In the indoor scenario, the RIS provided a $26 \mathrm{~dB}$ power gain at the receiver, even when the signal is blocked by a 30 $\mathrm{cm}$ thick concrete wall. In the short-range outdoor test, the RIS provided a $27 \mathrm{~dB}$ power gain. These gains are well aligned with theory and demonstrate the effectiveness of the proposed beamforming algorithm. We also carried out the world's first long-range outdoor field trial, over $500 \mathrm{~m}$. The RIS provided a $14 \mathrm{~dB}$ gain, which was sufficient to deliver $32 \mathrm{Mbps}$ using only $23 \mathrm{dBm}$ of transmit power. It should be noted that the power consumption of our RIS prototype is only about $1 \mathrm{~W}$. Our results demonstrated the great potential of RIS for future communication systems.

\section{ACKNOWLEDGMENT}

Discussions with Linglong Dai and Shi Jin are gratefully acknowledged.

\section{REFERENCES}

[1] T. L. Marzetta, "Noncooperative cellular wireless with unlimited numbers of base station antennas," IEEE Trans. Wireless Commun., vol. 9, no. 11 , pp. 3590-3600, Nov. 2010.

[2] E. G. Larsson, O. Edfors, F. Tufvesson, and T. L. Marzetta, "Massive MIMO for next generation wireless systems," IEEE Commun. Mag., vol. 52, no. 2, pp. 186-195, Feb. 2014.

[3] E. Björnson, J. Hoydis, and L. Sanguinetti, "Massive MIMO networks: Spectral, energy, and hardware efficiency," Found Trends ${ }^{\circledR}$ Signal Process., vol. 11, no. 3-4, pp. 154-655, 2017.

[4] H. Asplund, D. Astely, P. von Butovitsch, T. Chapman, M. Frenne, F. Ghasemzadeh, M. Hagström, B. Hogan, G. Jöngren, J. Karlsson, F. Kronestedt, and E. Larsson, Advanced Antenna Systems for 5G Network Deployments. Academic Press, 2020.

[5] M. Dohler and Y. Li, Cooperative Communications: Hardware, Channel and PHY. John Wiley \& Sons, 2010.

[6] C. Liaskos, S. Nie, A. Tsioliaridou, A. Pitsillides, S. Ioannidis, and I. Akyildiz, "A new wireless communication paradigm through softwarecontrolled metasurfaces," IEEE Commun. Mag., vol. 56, no. 9, pp. 162169, Sep. 2018. 
[7] M. D. Renzo et al., "Smart radio environments empowered by reconfigurable intelligent surfaces: How it works, state of research, and road ahead," IEEE J. Sel. Areas Commun., vol. 38, no. 11, pp. 2450-2525, Nov. 2020.

[8] E. Björnson, Ö. Özdogan, and E. G. Larsson, "Reconfigurable intelligent surfaces: Three myths and two critical questions," IEEE Commun. Mag., vol. 58, no. 12, pp. 90-96, Dec. 2020.

[9] Q. Wu, S. Zhang, B. Zheng, C. You, and R. Zhang, "Intelligent reflecting surface aided wireless communications: A tutorial," IEEE Trans. Commun., vol. 69, no. 5, pp. 3313-3351, 2021.

[10] C. Huang, A. Zappone, G. C. Alexandropoulos, M. Debbah, and C. Yuen, "Reconfigurable intelligent surfaces for energy efficiency in wireless communication," IEEE Trans. Wireless Commun., vol. 18, no. 8, pp. 4157-4170, Aug. 2019.

[11] Q. Wu and R. Zhang, "Intelligent reflecting surface enhanced wireless network via joint active and passive beamforming," IEEE Trans. Wireless Commun., vol. 18, no. 11, pp. 5394-5409, Nov. 2019.

[12] E. Björnson, H. Wymeersch, B. Matthiesen, P. Popovski, L. Sanguinetti, and E. de Carvalho, "Reconfigurable intelligent surfaces: A signal processing perspective with wireless applications," arXiv preprint arXiv:2102.00742, 2021.

[13] T. J. Cui, D. Smith, and R. P. Liu, Metamaterials: Theory, Design, and Applications. Springer, Jan. 2010

[14] T. J. Cui, S. Liu, and L. Zhang, "Information metamaterials and metasurfaces," J. Mater. Chem. C, vol. 5, no. 15, pp. 3644-3668, 2017.

[15] R. A. Shelby, D. R. Smith, and S. Schultz, "Experimental verification of a negative index of refraction," Science, vol. 292, no. 5514, pp. 77-79, 2001.

[16] Y. Liang, R. Long, Q. Zhang, J. Chen, H. V. Cheng, and H. Guo, "Large intelligent surface/antennas (LISA): Making reflective radios smart," $J$. Commun. Inf. Netw., vol. 4, no. 2, pp. 40-50, Jun. 2019.

[17] O. Tsilipakos et al., "Toward intelligent metasurfaces: The progress from globally tunable metasurfaces to software-defined metasurfaces with an embedded network of controllers," Adv. Opt. Mater, no. 2000783, Sep. 2020.

[18] Q. Wu and R. Zhang, "Beamforming optimization for wireless network aided by intelligent reflecting surface with discrete phase shifts," IEEE Trans. Commun., vol. 68, no. 3, pp. 1838-1851, Mar. 2020.

[19] W. Tang, X. Li, J. Y. Dai, S. Jin, Y. Zeng, Q. Cheng, and T. J. Cui, "Wireless communications with programmable metasurface: Transceiver design and experimental results," China Commun., 2019.

[20] W. Tang, J. Y. Dai, M. Chen, X. Li, Q. Cheng, S. Jin, K. K. Wong, and T. J. Cui, "Programmable metasurface-based RF chain-free 8PSK wireless transmitter," Electron. Lett., 2019.

[21] W. Tang, M. Z. Chen, J. Y. Dai, Y. Zeng, X. Zhao, S. Jin, Q. Cheng, and T. J. Cui, "Wireless communications with programmable metasurface: New paradigms, opportunities, and challenges on transceiver design," IEEE Wireless Commun., vol. 27, no. 2, pp. 180-187, Apr. 2020

[22] L. Dai, B. Wang, M. Wang, X. Yang, J. Tan, S. Bi, S. Xu, F. Yang, Z. Chen, M. D. Renzo, C.-B. Chae, and L. Hanzo, "Reconfigurable intelligent surface-based wireless communications: Antenna design, prototyping, and experimental results," IEEE Access, vol. 8, pp. 45913$45923,2020$.

[23] V. Arun and H. Balakrishnan, "RFocus: Beamforming using thousands of passive antennas," in 17th USENIX Symposium on Networked Systems Design and Implementation (NSDI 20). USENIX Association, Feb. 2020, pp. 1047-1061.

[24] O. Ozdemir, F. Erden, I. Guvenc, T. Yekan, and T. Zarian, " $28 \mathrm{GHz}$ mmWave channel measurements: A comparison of horn and phased array antennas and coverage enhancement using passive and active repeaters," arXiv preprint arXiv:2002.00121, 2020.

[25] J. Y. Dai, W. K. Tang, J. Zhao, X. Li, Q. Cheng, J. C. Ke, M. Z. Chen, S. Jin, and T. J. Cui, "Wireless communications through a simplified architecture based on time-domain digital coding metasurface," Adv Mater. Technol., vol. 4, no. 7, p. 1900044, 2019.

[26] Skyworks Solutions, Inc., "SMV2019 to SMV2023 series: Hyperabrupt junction tuning varactors," SMV2019-079LF Datasheet, Aug. 2015.

[27] X. Tan, Z. Sun, J. M. Jornet, and D. Pados, "Increasing indoor spectrum sharing capacity using smart reflect-array," in 2016 IEEE International Conference on Communications (ICC), May. 2016, pp. 1-6.

[28] H. Yang, F. Yang, S. Xu, M. Li, X. Cao, J. Gao, and Y. Zheng, "A study of phase quantization effects for reconfigurable reflectarray antennas," IEEE Antennas Wireless Propag. Lett., vol. 16, pp. 302-305, Feb. 2017.

[29] H. Liu, X. Yuan, and Y.-J. A. Zhang, "Matrix-calibration-based cascaded channel estimation for reconfigurable intelligent surface assisted multiuser MIMO," IEEE J. Sel. Areas Commun., vol. 38, no. 11, pp. 2621-2636, Nov. 2020
[30] L. Wei, C. Huang, G. C. Alexandropoulos, and C. Yuen, "Parallel factor decomposition channel estimation in RIS-assisted multi-user MISO communication," in 2020 IEEE 11th Sensor Array and Multichannel Signal Processing Workshop (SAM), Jun. 2020, pp. 1-5.

[31] A. Taha, M. Alrabeiah, and A. Alkhateeb, "Enabling large intelligent surfaces with compressive sensing and deep learning," CoRR, vol. abs/1904.10136, 2019.

[32] B. Zheng and R. Zhang, "Intelligent reflecting surface-enhanced OFDM: Channel estimation and reflection optimization," IEEE Wireless Commun. Lett., vol. 9, no. 4, pp. 518-522, Apr. 2020.

[33] Z. Wang, L. Liu, and S. Cui, "Channel estimation for intelligent reflecting surface assisted multiuser communications: Framework, algorithms, and analysis," IEEE Trans. Wireless Commun., vol. 19, no. 10, pp. 66076620 , Oct. 2020.

[34] E. Björnson and L. Sanguinetti, "Power scaling laws and near-field behaviors of massive MIMO and intelligent reflecting surfaces," IEEE Open J. Commun. Soc., vol. 1, pp. 1306-1324, 2020.

[35] 3GPP, Study on Channel Model for Frequencies from 0.5 to 100 $\mathrm{GHz}$ (Release 15). Technical Report TR 38.901, available: http://www.3gpp.org, 2019.

[36] H. Yin, H. Wang, Y. Liu, and D. Gesbert, "Addressing the curse of mobility in massive MIMO with Prony-based angular-delay domain channel predictions," IEEE J. Sel. Areas Commun., vol. 38, no. 12, pp. 2903-2917, Dec. 2020.

[37] H. Yin, D. Gesbert, M. Filippou, and Y. Liu, "A coordinated approach to channel estimation in large-scale multiple-antenna systems," IEEE J. Sel. Areas Commun., vol. 31, no. 2, pp. 264-273, Feb. 2013.

[38] A. Adhikary, J. Nam, J.-Y. Ahn, and G. Caire, "Joint spatial division and multiplexing - the large-scale array regime," IEEE Trans. Inf. Theory, vol. 59, no. 10, pp. 6441-6463, Oct. 2013

[39] W. Tang, M. Z. Chen, X. Chen, J. Y. Dai, Y. Han, M. Di Renzo, Y. Zeng, S. Jin, Q. Cheng, and T. J. Cui, "Wireless communications with reconfigurable intelligent surface: Path loss modeling and experimental measurement," IEEE Trans. Wireless Commun., vol. 20, no. 1, pp. 421439, Jan. 2021.

[40] X. Zhang, Q. Li, F. Liu, M. Qiu, S. Sun, Q. He, and L. Zhou, "Controlling angular dispersions in optical metasurfaces," Light: Sci. Appl., vol. 9, no. 76, p. 76, May. 2020.

[41] K. Ntontin, A.-A. A. Boulogeorgos, D. G. Selimis, F. I. Lazarakis, A. Alexiou, and S. Chatzinotas, "Reconfigurable intelligent surface optimal placement in millimeter-wave networks," IEEE Open J. Commun. Soc., vol. 2, pp. 704-718, Mar. 2021.

[42] Z. Wang, L. Tan, H. Yin, W. Kai, X. Pei, and D. Gesbert, "A received power model for reconfigurable intelligent surface and measurementbased validations," in IEEE 22nd Workshop on Signal Processing Advances in Wireless Communications, SPAWC 2021, Lucca, Italy, Sept. 2021, pp. 1-5. 